\title{
Editorial
}

\section{Toward a Better Understanding and Evidence-Based Intervention for Early Trauma Responses}

\author{
Birgit Kleim
}

Department of Psychology, University of Zurich, Switzerland

In today's turbulent world, exposure to stress and traumatic events, such as road traffic accidents, interpersonal violence, or natural disasters, are common. The majority of individuals will be exposed to at least one traumatic event in the course of their life. Some, such as refugees, asylum seekers, or other forcefully displaced individuals are often exposed to multiple traumatic events in the context of war, persecution, and displacement. Some professionals, for example, emergency first responders, will also be routinely exposed to traumatic stressors as part of their profession. A key finding from mental health and resilience research is that trauma affects everyone differently. While trauma exposure is common, psychiatric symptoms and disorders in response to such events are, fortunately, rare (Galatzer-Levy et al., 2018). Disorders, such as acute or posttraumatic stress disorder (PTSD), depression, or adjustment disorder are, however, pervasive and often follow a chronic course (Zlotnick et al., 1999). A better understanding of early responses, underpinning mechanisms, and the possibility to forecast who will develop symptoms of mental disorders and who is resilient is crucial (Shalev et al., 2019). Such information will help pave the way to advance the prevention and intervention science and shape and advise practice and policy makers in the context of acute trauma.

Numerous informative features and processes of interest can be captured during the acute phase post-trauma. These range from basic medical and clinical variables, psychiatric symptoms of acute stress, arousal, memory, and emotion regulation, to social and interpersonal processes. This time window in the early aftermath of trauma, that is, hours and days post-exposure, is crucial (Squire et al., 2015; Monfils \& Holmes, 2018). Many dysfunctional, as well as benign processes, are initiated and unfold during this phase. More importantly, perhaps, this is often the only period during which many trauma survivors are in contact with professionals, such as the police, paramedics, legal authorities, mental health professionals, and others (Schultebraucks \& Chang, 2020). It is thus a vital time that ought to be exploited to collect relevant data and informative features that may help answer the question of who develops chronic disorders later, as well as who is resilient. In this special section of European Psychologist, key articles focus on the early period after trauma exposure.

In the first contribution, Azza and colleagues (2020) shed light on potential mechanisms underpinning the development of intrusive memories, a core feature of PTSD (Bryant et al., 2017). Specifically, the authors review literature on sleep and memory formation and re-consolidation processes early, that is, hours to days, after trauma exposure. Based on experimental laboratory and naturalistic studies, it is argued that sleep may protect from developing intrusive memories, possibly by affective depotentiation and integration of newly formed trauma memories. Despite the fact that the actual features of the sleep architecture supporting these processes are still in need of more rigorous research and replication, these findings support the role of sleep in preventing intrusive trauma memories and PTSD in trauma survivors.

The second paper by Wild and colleagues (2020) provides a review on non-pharmacological interventions to improve well-being, resilience, or stress management in first responders, a population exposed to significant trauma as part of their profession and therefore at specific risk to develop PTSD and other stress-related disorders. Based on 13 studies, the authors report large effects for interventions that targeted modifiable risk factors for trauma-related psychiatric disorders, such as exercise and imagery interventions. Targeting modifiable predictors of trauma-related 
psychiatric disorders through training may protect the health of first responders and others who routinely face trauma in their line of work and could be applied early post-exposure to prevent PTSD.

The third contribution by Malgaroli and Schultebraucks (2020) provides the readers of this section with a methodological key to advance our ability to forecast chronic PTSD from acute stress responses. The authors provide a brief overview of artificial intelligence methods, along with an overview of the emerging literature on computational approaches to PTSD research. Their discussion of emerging clinical applications brought about by large-scale data-driven analytics and sensitive and precise forecasting rounds up this section by outlining the potential of fresh computational approaches for personalized treatment in the context of acute stress and PTSD and the possibility to forecast who develops chronic PTSD from acute stress responses.

Taken together, the papers comprised in this section provide an update and advice on preventing and treating stress- and trauma-related disorders, for example, PTSD, with a specific focus on acute stress responses and features characterizing the period early after trauma. As such, they aim to identify key opportunities for trauma professionals and professional societies to increase ways of intervening and ameliorating negative (mental) health consequences of trauma.

\section{References}

Azza, Y., Wilhelm, I., \& Kleim, B. (2020). Sleep early after trauma: A target for prevention and early intervention for posstraumatic stress disorder? European Psychologist, 25(4), 239-251. https://doi.org/10.1027/1016-9040/a000401

Bryant, R. A., Creamer, M., O'Donnell, M., McFarlane, A., Silove, D., \& Hadzi-Pavlivic, D. (2017). Acute and chronic posttraumatic stress symptoms in the emergence of porsstraumatic stress disorder. JAMA Psychiatry, 74(2), 135-142. https://doi.org/ 10.1001/jamapsychiatry.2016.3470

Galatzer-Levy, I. R., Huang, S. H., \& Bonanno, G. A. (2018). Trajectories of resilience and dysfunction following potential trauma: A review and statistical evaluation. Clinical Psychology Reviews, 63, 41-55. https://doi.org/10.1016/j.cpr.2018.05.008

Malgaroli, M., \& Schultebraucks, K. (2020). Artificial intelligence and posttraumatic stress disorder (PTSD): An overview of advances in research and emerging clinical applications.
European Psychologist, 25(4), 272-282. https://doi.org/ $10.1027 / 1016-9040 / a 000423$

Monfils, M. H., \& Holmes, E. A. (2018). Memory boundaries: Opening a window inspired by reconsolidation to treat anxiety, traumarelated, and addiction disorders. Lancet Psychiatry, 5(12), 1032-1042. https://doi.org/10.1016/S2215-0366(18)30270-0

Schultebraucks, K., \& Chang, B. P. (2020). The opportunities and challenges of machine learning in the acute care setting for precision prevention of posttraumatic stress sequelae. Experimental Neurology, 4, 113526. https://doi.org/10.1016/j.expneurol. 2020.113526

Shalev, A. Y., Gevonden, M., Ratanatharathorn, A., Laska, E., van der Mei, W. F., Qi, W., Lowe, S., Lai, B. S., Bryant, R. A., Delahanty, D., Matsuoka, Y. J., Olff, M., Schnyder, U., Seedat, S., deRoonCassini, T. A., Kessler, R. C., \& Koenen, K. C., International Consortium to Predict PTSD. (2019). Estimating the risk of PTSD in recent trauma survivors: Results of the International Consortium to Predict PTSD (ICPP). World Psychiatry, 18(1), 77-87. https://doi.org/10.1002/wps.20608

Squire, L. R., Genzel, L., Wixted, J. T., \& Morris, R. G. (2015). Memory consolidation. Perspectives in Biology, 7(8), a021766. https://doi.org/10.1101/cshperspect.a021766

Wild, J., El-Salahi, S., \& Degli Esposti, M. (2020). The effectiveness of interventions aimed at improving well-being and resilience to stress in first responders: A systematic review. European Psychologist, 25(4), 252-271. https://doi.org/10.1027/10169040/a000402

Zlotnick, C., Warshaw, M., Shea, M. T., Allsworth, J., Pearlstein, T., \& Keller, M. B. (1999). Chronicity in posttraumatic stress disorder (PTSD) and predictors of course of comorbid PTSD in patients with anxiety disorders. Journal of Trauma Stress, 12(1), 89-100. https://doi.org/10.1023/A:1024746316245

Published online January 19, 2021

\section{Birgit Kleim}

Department of Psychology

University of Zurich

Binzmühlestrasse 14, Box 8

8050 Zurich

Switzerland

birgit.kleim@uzh.ch

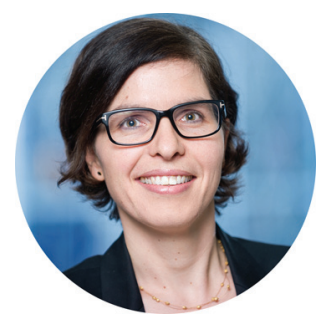

Birgit Kleim (PhD) is professor of Experimental Psychopathology and Psychotherapy. She earned her PhD from King's College London before joining the University of Zurich in 2010. Her main interests include experimental investigation of anxiety and PTSD, including investigation of early risk and protective factors, such as sleep. 